Linguistique, littérature, didactique

\title{
La règle et l'exception selon Ferdinand Brunot
}

Rules and Exceptions according to Ferdinand Brunot

\section{Aurélia Elalouf}

\section{(2) OpenEdition}

\section{Journals}

Édition électronique

URL : http://journals.openedition.org/pratiques/2630

DOI : 10.4000/pratiques.2630

ISSN : 2425-2042

\section{Éditeur}

Centre de recherche sur les médiations (CREM)

\section{Référence électronique}

Aurélia Elalouf, « La règle et l'exception selon Ferdinand Brunot », Pratiques [En ligne], 167-168 | 2015, mis en ligne le 01 avril 2016, consulté le 14 novembre 2019. URL : http://journals.openedition.org/ pratiques/2630; DOI : 10.4000/pratiques.2630

Ce document a été généré automatiquement le 14 novembre 2019.

(c) Tous droits réservés 


\title{
La règle et l'exception selon Ferdinand Brunot
}

\author{
Rules and Exceptions according to Ferdinand Brunot
}

\author{
Aurélia Elalouf
}

1 Le présent article est une contribution à l'analyse historique et épistémologique des travaux de F. Brunot (1860-1938), qui se situe dans le prolongement des réflexions entre autres - de J.-C. Chevalier (1991) et H. Besse (1995). Il s'agit des premiers éléments d'une recherche dans laquelle nous nous intéressons en particulier aux considérations que F. Brunot développe dans La Pensée et la langue (1922), ouvrage qui constitue le fruit de presque 20 ans de réflexion, tant sur l'enseignement de la grammaire que sur l'histoire de la langue, et dont le statut est ambivalent, comme l'atteste cette déclaration de l'auteur dans la préface :

"[Ce livre n'est pas] une "Grammaire". Sans doute on y retrouvera les mots d'adjectifs, de verbes, d'adverbes, ainsi de suite. On y retrouvera aussi les règles qui régissent les variations des mots et leur agencement. J'ai fait la critique de plusieurs de ces règles, je n'en ai écarté aucune, me bornant à mettre mon lecteur à même de distinguer celles qui ont une autorité véritable. Mais mon but n'a pas été de donner une grammaire revue et corrigée » (Brunot, 1922 : VII).

Le point qui nous intéresse ici est la mention que F. Brunot fait des «règles" de grammaire, qui constituent pour lui, avec la terminologie ("adjectifs", "verbes", «adverbes »), un élément définitoire du genre grammatical et qui, pour cette raison même, sont sujettes à discussion dès lors que l'on adopte, comme il le fait, une posture de critique à l'égard d'une certaine manière de faire de la grammaire, d'une certaine manière de décrire et d'enseigner la langue. On peut ainsi s'interroger sur les différents aspects de la réflexion de $\mathrm{F}$. Brunot sur la notion de "règle grammaticale " ainsi que sur les incidences de cette réflexion sur sa pratique de grammairien dans La Pensée et la langue. Or, il apparait rapidement à l'analyse que la critique des règles menée par F. Brunot ne peut être dissociée d'une remise en cause du statut des "exceptions", c'est-à-dire des formes linguistiques qui sont jugées valides par le grammairien mais qui dérogent à la règle - obligeant à restreindre la portée de cette dernière ou à en revoir la formulation ${ }^{1}$. Notre étude repose sur une analyse des collocations dans 
lesquelles apparaissent les termes règle et exception (et les termes qui leur sont associés) dans le métadiscours de F. Brunot ${ }^{2}$. Nous traiterons tout d'abord des défauts dont souffrent, selon lui, la plupart des règles de grammaire établies par ses prédécesseurs, et des solutions qu'il adopte dans La Pensée et la langue pour reformuler ces "fausses règles " et se débarrasser de leurs exceptions. Puis nous essaierons de cerner ce qui définit pour F. Brunot une "vraie règle » de grammaire, avant de tracer des pistes de travail pour la poursuite de la recherche.

\section{La critique des règles de la « grammaire dogmatique »}

3 La mise en place de l'instruction primaire gratuite, laïque et obligatoire (lois Jules Ferry de 1881 et 1882) constitue pour F. Brunot un enjeu de politique linguistique considérable, qui le conduit à manifester un intérêt constant pour les questions d'enseignement de la langue et de la grammaire. C'est dans cette perspective qu'il se positionne en faveur de la réforme de l'orthographe de 1905-1906, participe à l'élaboration de la nomenclature grammaticale de 1910 et élabore, avec N. Bony, les trois livres de la Méthode de langue française (publiés entre 1905 et 1911). Ces différentes expériences le poussent à tenir un discours de plus en plus critique sur ce qu'il appelle la "grammaire dogmatique» (Brunot, 1922: XIII), terme qui lui permet à la fois de condamner une partie des descriptions des grammairiens et remarqueurs du XVII ${ }^{\mathrm{e}}$ siècle, de récuser l'héritage de la grammaire générale et de dénoncer les excès de la grammaire scolaire. Les règles grammaticales établies par ses prédécesseurs, tour à tour qualifiées d'" extravagantes", d'" arbitraires », d'" artificielles", etc., et entrainant l'énumération sans fin d'" exceptions et de sous-exceptions", constituent l'une des ses cibles privilégiées. La critique de F. Brunot porte à la fois sur le contenu de connaissance de ces règles et sur leur mise en œuvre à l'école : «Il n'y a, dit-il, qu'à feuilleter quelques pages d'un manuel pour trouver des spécimens variés de ces fautes contre la raison, la vérité et la pédagogie » (Brunot, 1909 : 3).

\subsection{La critique des règles d'un point de vue pédagogique}

4 Les observations de F. Brunot concernent en premier lieu l'enseignement des règles nécessaires à l'apprentissage de la lecture et de l'écriture à l'école primaire. Pour le jeune élève, ces règles, telles qu'elles sont habituellement formulées, sont très difficiles à comprendre. Ainsi, la règle de formation du féminin des adjectifs qualificatifs, selon laquelle « le féminin se forme par l'addition d'un $e$ muet », en entretenant l'ambigüité entre les plans oral et écrit, ne manque pas de déconcerter :

«Lorsque l'enfant applique cette règle à des adjectifs tels que mûr, égal: un fruit mûr, une pomme mûre, etc., comme les deux formes de l'adjectif se prononcent de même, l'enfant comprend à peu près. On a ajouté un $e$ muet, c'est-à-dire rien, et il entend, en effet, un féminin mûre semblable au masculin mûr. Jusque-là, tout va bien.

Mais dès qu'il écoute petit et petite, gros et grosse, grand et grande, etc., il entend au féminin des consonnes qui ne se prononçaient pas au masculin, et il se trouve en présence de ce mystère : En ajoutant un $e$ muet, qui n'est rien, qui serait tout au plus une voyelle, on entend toutes les consonnes de l'alphabet!» (Brunot, 1909: 104-105, nous soulignons). 
Un autre exemple est celui de la règle du pluriel des noms propres, qui, ne considérant les faits que sous le seul prisme de l'orthographe, va à contresens des intuitions de l'élève :

"Un chapitre du "nombre dans le nom" ne manque point de donner une liste des mots qui ne s'emploient pas au pluriel. Ce sont d'abord les noms propres de personnes, "qui ne sortent pas du singulier", sauf quelques-uns. Or, il suffit que l'enfant entende parler d'une famille quelconque du village, des Cuny, des Dupont, des Demange, pour qu'il ne comprenne plus. Puisqu'on dit "les Cuny" et que les Cuny sont cinq, comment ne serait-ce pas le pluriel? Qu'on mette une $s$ ou non, cela ne fait rien à l'affaire. On confond ici le pluriel et la marque du pluriel» (Brunot, 1909 : 108 , nous soulignons).

De ce fait, les règles ne sont pour l'élève, et pour le maitre lui-même, que de «vaine[s] formule[s]» (Brunot, 1909: 111) à apprendre par cœur. Pour retenir les exceptions orthographiques en particulier, «l'enfant [est] réduit à écrire, à copier des listes sans aucun intérêt, des listes de pluriels, des listes de temps, de formes verbales ou autres " (Brunot, 1913a: 127), ce qui demande un effort considérable et ne profite même pas à « un développement rationnel de la mémoire » (ibid.). Enfin, les règles présentent de nombreuses difficultés d'application pour les élèves, encore accrues par l'inadéquation des exercices proposés, comme ces «exercices mécaniques» (Brunot, 1920:161) qui contraignent les élèves à des manipulations formelles d'une abstraction dont, selon F. Brunot (1913a : 127), « un cerveau d'enfant est à peu près incapable ». Un exemple est la règle d'accord du participe passé des verbes « accidentellement pronominaux » :

«On enseigne à faire accorder le participe passé des verbes pronominaux comme si ces verbes étaient conjugués avec avoir. Pour les "accidentellement pronominaux" la règle est : Il y a accord quand le complément direct précède le participe. L'enfant doit se demander si le complément pronominal est un complément direct ou indirect. Ex. : Nous nous sommes attaqués à plus fort que nous".

«Pour savoir s'il faut une $s$ à attaqués, puisque s'attaquer n'est pas un verbe "essentiellement pronominal", on considère le complément nous. Voilà l'enfant obligé d'examiner si nous nous sommes attaqués à... correspond à : nous avons attaqué à nous à... ou bien à : nous avons attaqué nous à plus fort que nous; il a à choisir entre deux phrases qui n'offrent ni l'une ni l'autre aucun sens " (Brunot, 1909: 5, nous soulignons).

7 Ainsi, pour beaucoup de maitres, et même de professeurs du secondaire, l'enseignement de la grammaire, tel qu'il est pratiqué, est « inutile », voire "funeste » (Brunot, 1909: 2). En effet, cet enseignement n'a pas ou que peu d'incidence positive sur l'emploi que l'élève fait de sa langue :

«Sans doute les enfants étudient et récitent leurs leçons, mais machinalement : ces règles abstraites, encombrées d'exceptions et de sous-exceptions, ne pénètrent pas réellement leur esprit; ils font, tant bien que mal, les devoirs écrits qui accompagnent ces règles dans leurs livres; mais on ne constate guère que cette étude, qui leur coûte tant de peine, ait une influence profonde, soit sur leur langage, soit sur leurs rédactions; des exercices immédiats d'application la correction ne s'étend pas à leur usage quotidien, comme elle le devrait » (Brunot, $1909: 1$, nous soulignons).

8 Davantage, cet enseignement tend à fausser le rapport des élèves à la langue : "En parlant suivant ces théories, dit F. Brunot, on se ferait moquer de soi, comme ces enfants formés pour la dictée qui prononcent toutes les lettres - j'en ai entendu - dans : les poules couvent!» (Brunot, 1922: IX). Selon lui, le primat accordé à l'orthographe constitue le «fléau véritable» (Brunot, $1920: 161)$ de cet enseignement ${ }^{3}$, car il oblige le 
maitre à "habituer son élève à accepter sans répugnance les choses les plus incompréhensibles ou les plus visiblement absurdes, et cela est, ajoute-t-il, abêtissant et contre-éducatif » (Brunot, 1909: 30). Dès lors, l'objectif poursuivi par F. Brunot est de :

«Montrer qu'en grammaire il n'existe point de dogmes qu'on doive recevoir sans comprendre, et accepter comme des vérités surnaturelles [...]. De la sorte, la pédagogie grammaticale se retrouve en harmonie avec la pédagogie générale de l'école laïque, qui veut former les esprits à la réflexion et au libre examen » (Brunot, 1909:125, nous soulignons).

\subsection{La critique des règles d'un point de vue épistémologique}

9 La remise en cause de l'existence de tels «dogmes" impose de prendre en considération parallèlement l'histoire de la langue et de sa description, l'évolution des usages et la constitution des règles grammaticales. L'enjeu des critiques formulées par F. Brunot est de discuter la pertinence de l'action que les grammairiens ont exercé sur la langue au cours du temps, en posant des règles qui, pour un grand nombre d'entre elles, ne satisfont pas aux exigences de scientificité qu'il réclame. Les principaux reproches qu'il adresse aux « faiseurs de règles » et autres « législateurs » sont d'avoir observé et décrit avec trop peu d'attention les faits linguistiques et d'être souvent allé, par l'inadéquation de leur description, à l'encontre de (ce qu'il considère devoir être) l'évolution « naturelle » de la langue.

Beaucoup de grammairiens auraient ainsi formulé des « règles contraires à la vérité des faits » (Brunot, $1909: 4$ 4-5). F. Brunot (1922: 589) considère par exemple que la règle de formation du féminin des adjectifs qualificatifs (règle déjà mentionnée supra) est une « règle tout à fait insuffisante » puisqu'elle ne s'attache pas à décrire la «réalité linguistique " (comprendre ici : "la langue parlée»). D'autres règles sont "si étroites qu'elles sont à chaque instant violées par l'usage » (Brunot, 1909: 108), c'est-à-dire qu'elles ne permettraient pas de décrire les réalisations en discours. Les règles selon lesquelles les noms de matière et les noms abstraits n'ont pas de pluriel sont ainsi qualifiées d'«arbitraires " (ibid.), ce qui signifie, dans le métalangage de F. Brunot, qu'elles sont le produit de la seule «fantaisie » des grammairiens, que rien dans l'usage ne permet de les établir. Au chapitre consacré à «la notion de nombre », F. Brunot (1922: 97) soutient que « toutes les idées à peu près sont susceptibles d'être conçues au pluriel ». En effet, même si, " par définition, les choses non nombrables n'ont pas de pluriel ", d'une part « la plupart des matières existent en plusieurs espèces » (le cours des aciers), "d'autre part les objets faits avec les matières portent le nom de ces matières " (des zincs d'art) (ibid. : 96). De la même manière, les idées abstraites peuvent se décliner en plusieurs espèces (avoir toutes les ambitions), et les actes qu'elles inspirent sont souvent désignés par les mot utilisés pour nommer ces mêmes idées (avoir des bontés pour quelqu'un). Quoique les observations de $\mathrm{F}$. Brunot soient ici très allusives, on peut penser qu'il accuse les auteurs de ces "fausse[s] règle[s] au sujet des pluriels » (Brunot, 1909: 109) non pas d'avoir ignoré les cas où l'on rencontre des noms de matière ou des noms abstraits au pluriel ${ }^{4}$, mais plutôt d'avoir mis sur deux plans distincts les emplois massifs et comptables de ces noms, d'avoir considéré comme premiers les emplois massifs (qui tiendraient à la valeur même de ces noms en langue) et comme secondaires, exceptionnels les emplois comptables (qui ne seraient que des réalisations possibles en discours). 
Autre défaut mentionné par F. Brunot, les grammairiens auraient fixé des règles sans prêter attention aux changements linguistiques en cours et à (ce qu'on appellerait) la cohérence interne de la langue, soit que ces grammairiens ignorent l'histoire de la langue, soit qu'ils n'aient pas jugé nécessaire de la prendre en considération. Un exemple est la règle qui fixe l'emploi de la formule c'est. En moyen français, remarque F. Brunot, deux formes concurrentes existaient : il était tout autant possible de dire « ce suis-je, c'es-tu» (formes de l'ancien français) que «c'est moi, c'est toi » (formes modernes), concurrence qui résulte du changement de fonction accordée à ce, au départ attribut, puis "peu à peu considéré comme le sujet, d'où une tendance à accorder le verbe avec lui : c'est vous, et à substituer moi, toi, à je, tu» (Brunot, 1922 : $287)^{5}$. Au XVI ${ }^{e}$ siècle, le verbe ne varie plus qu'avec la troisième personne du pluriel (concurrence entre c'est eux et ce sont eux). Or, selon F. Brunot, si «la langue avait été laissée à elle-même, elle aurait fini par ne plus connaître, au bout d'un temps plus ou moins long, que le seul c'est» (ibid.). Ainsi, la règle fixée par les grammairiens du XVII selon laquelle, "par exception, si le verbe être tombe sur un substantif ou sur un pronom de la troisième personne du pluriel, il peut en recevoir l'accord» (ibid.) apparait pour F. Brunot triplement condamnable :

- la règle a entravé l'évolution de la langue ;

- elle repose sur une analyse syntaxique qui ne peut convenir à la langue moderne : ce est considéré comme un attribut dans la phrase : ce furent elles qui l'aidèrent, « d'après l'analyse officielle » qui reconstruit : elles qui l'aidèrent furent ce (dont il s'agit) (1922 : 288) ;

- elle justifie la conservation de deux formes concurrentes par l'existence d'une nuance de sens qui n'a pas lieu d'être : selon Lemaire, « avec le verbe au pluriel, on porte l'attention sur le mot qui commande l'accord» (ce sont les grands qui ont donné du crédit à l'impie), alors qu'«avec le singulier, on laisse au démonstratif toute sa portée, et c'est sur l'idée, sur l'action même qu'il attire la réflexion » (c'est eux qu'il faut récompenser) (cité par F. Brunot, ibid.).

D'où F. Brunot conclut :

«La courte histoire qui précède suffit à faire juger la valeur d'une doctrine fondée sur une ignorance totale de la marche du langage. Nous avons là le type même de la distinction imaginaire, intervenue au moment où de soi-même tout se clarifiait et s'unifiait, c'est devenant une formule invariable» (Brunot, 1922: 288, nous soulignons).

11 De manière générale, F. Brunot condamne toutes les règles qui ont participé au maintien ou à l'introduction de contradictions et de complications inutiles dans la langue : "C'est une règle bien dangereuse que celle qui oppose des coupe-papier à des ouvre-lettres", note F. Brunot (1922: 106) dans un passage consacré au pluriel orthographique des noms composés. "Des règles capricieuses" (ibid. : 425) régissent l'emploi de l'article devant les noms propres de lieu, remarque-t-il encore :

«Pourquoi de France et de la Chine ? [...] Dans le cas particulier, la règle a été généralisée : en France, en Chine. Malheureusement loin qu'elle le fût partout, la langue n'a plus jamais échappé aux contradictions : aller en Meurthe-et-Moselle, dans le Doubs; habiter dans la Meuse, en Corrèze » (Brunot, 1922 : 169, nous soulignons).

12 L'exemple emblématique reste la règle de l'accord de tout proposée par C. F. de Vaugelas, qui fait dire tout criminels qu'ils sont mais toutes criminelles qu'elles sont et écrire une rue toute pavée mais une rue tout entière : pour F. Brunot (1921a : 38), cette règle n'est qu'une « invention médiocre d'hommes qui sont tombés dans d'inextricables contradictions, faute de savoir et d'esprit d'observation ». 
À l'inverse, certains grammairiens sont allés à l'encontre de la "réalité linguistique " en participant, au nom de la logique, à un appauvrissement de la langue. F. Brunot (1909: 50) pense ici à tous les auteurs qui se placent dans le sillage de la grammaire générale, auxquels on doit « des définitions et des règles abstraites, dont on a retiré tout ce qui était vivant». Sa critique concerne en premier lieu le fait que ces grammairiens auraient porté un intérêt trop exclusif aux formes, au détriment du sens. $\mathrm{Au}$ chapitre intitulé "Les fausses règles et la réalité », F. Brunot (1922: 780) fait remarquer que «c'est le sens même qui borne les possibilités » d'emploi des temps dans une proposition subordonnée "et non la grammaire». Le "principe» sur lequel s'appuie les règles de concordance des temps est donc "mauvais» (ibid.: 782), puisqu' " on a substitué la pauvre mécanique [de ces règles], toute extérieure et formelle, aux rapports véritables de la chronologie » (ibid. : X). La critique de F. Brunot porte en second lieu sur le désir excessif de régularité manifesté par ces grammairiens, qui n'observent pas les faits "tels que la vie de la langue les présente », mais les analysent «en les rapportant à une langue idéale, construite de toutes pièces » (ibid. : 40). Le recours à l'ellipse constitue ainsi pour F. Brunot un subterfuge autorisant " toutes les fantaisies d'analyse ", à l'instar de l'analyse de la proposition des Messieurs de Port Royal, que l'on retrouve jusque dans les grammaires scolaires du xix siècle: «Un seul verbe exprime l'affirmation, c'est être; tout autre verbe renferme en lui le verbe être; toute proposition doit être ramenée à trois termes, en sous-entendant ceux qu'il faut, et, au besoin, en les sous-entendant tous " (Brunot, 1909: 43-44). Plus généralement, F. Brunot (1922: 641) critique tous les cas où les grammairiens ont cherché à soumettre la langue à des règles "trop rigoureuses" au point de devoir, "pour faire cadrer les faits avec les principes, imaginer parfois des explications déconcertantes ». Un exemple est celui de la place de l'adjectif épithète par rapport au nom qu'il qualifie :

«Dans la locution: à haute voix, l'adjectif, dit-on, peut-être considéré comme essentiel. Le mot voix, par lui-même, implique une certaine élévation que l'adjectif haute ne fait qu'amplifier, comme le ferait un suffixe augmentatif. (!?) Au contraire dans la locution à voix basse, la qualité basse n'est qu'accidentelle, exceptionnelle : D'où l'ordre adopté. Mais ne dit-on pas : à voix haute? (Brunot, 1922: 641, nous soulignons).

Selon F. Brunot (1922: 642), le tort de ces grammairiens a été de vouloir édicter des règles de syntaxe sans distinguer les cas où il est effectivement « impossible de donner telle place à l'adjectif » de ceux, «beaucoup plus nombreux, où cela est simplement contraire à l'usage ordinaire ", inhabituel, mais néanmoins possible (pour des raisons stylistiques notamment), et pour lesquels on ne peut donc établir de « règle rigide ». Un autre exemple de tentative de simplification abusive de la langue concerne l'emploi de chacun, qui a donné lieu à "des polémiques sans fin entre les grammairiens » (ibid.: 131). Certains " théoriciens » ont ainsi soutenu qu'il n'est pas acceptable d'employer les possessifs notre, votre, leur avec le distributif chacun (les enfants ont mangé chacun leur pomme), car, « du moment que vous diviser l'action d'ensemble [...], vous la considérez comme formant autant d'actions de détail, donc vous devez pour chacune employer le terme qui convient à une et non à plusieurs (ibid. : 131-132). F. Brunot soutient que de telles considérations n'ont pas lieu d'être et qu'elles conduisent à se priver de la possibilité d'exprimer une nuance sémantique :

«Avec chacun, qu'est-ce qui convient, le possessif de l'unité ou celui de la pluralité?

[...] doit-on dire : les hommes ont mis chacun son chapeau, ou : ils ont mis chacun leur

chapeau? Les uns ont tenu pour son chapeau, les autres pour leur chapeau; on 
invoquait de prétendus principes logiques. En réalité il n'y a pas de logique làdedans, il y a deux façons de considérer une même action accomplie par une collectivité.

(...) Treize cavaliers attendent, et il n'y a que douze chevaux, ils n'ont pas chacun son cheval. Chacun leur cheval signifierait plutôt qu'ils n'ont pas chacun le cheval qu'ils ont l'habitude de monter » (Brunot, 1922 : 131-132) [nous soulignons].

\section{La réécriture des règles dans La Pensée et la langue} règles de la "grammaire dogmatique». L'ouvrage n'est pas (ou pas seulement) «ce Manuel des fausses règles» que F. Brunot (1922: XI) appelle de ses vœux et qui, dit-il, «deviendra bientôt un bréviaire pédagogique, et un livre d'affranchissement national ». Ses réflexions l'amènent à plusieurs reprises à proposer de nouvelles règles, ou tout au moins à esquisser quelques possibilités de reformulation des règles orthographiques, morphologiques et syntaxiques existantes.

\subsection{Règles orthographiques}

L'orthographe étant une simple convention d'écriture, il est (en principe) possible d'en modifier les règles sans modifier la langue elle-même. Suite à l'échec de la réforme orthographique de 1905-1906, on ne trouve que très peu de traces des propositions de la Commission dans La Pensée et la langue. Deux cas se présentent. Dans le premier cas, il est simplement fait allusion à la possibilité d'une modification des règles. Alors que dans le rapport de la Commission est posée la règle selon laquelle « $x$ cessera d'être employée à la place de $s$ à la fin des mots » (Brunot, 1905 : 384), F. Brunot se contente, dans La Pensée et la langue, de la suggérer :

«Quant à l'x, elle est toujours de règle (sauf dans le mot landaus, qui étant étranger, a échappé à la règle). On n'en voit pas bien l'utilité. Pourquoi cadeaux avec $x$ ? A quoi bon feux et pneus? poux et fous? Un acte de courage nous débarrasserait d'une complication absurde et inutile (1).

(1) Il n'y a que quelques mots en oux: bijoux, cailloux, choux, genoux, joujoux, hiboux, poux » (Brunot, 1922 : 104, nous soulignons).

Ce passage, aussi anecdotique qu'il puisse paraitre, est caractéristique de la pratique grammaticale de F. Brunot dans La Pensée et la langue : il ne s'agit pas tant de rappeler les règles traditionnelles (on remarque que la liste des sept pluriels en -oux du français est reléguée en note de bas de pages) que d'en discuter le bien-fondé.

Le second cas qui peut se présenter est le suivant: à défaut de pouvoir modifier l'orthographe en vigueur, $\mathrm{F}$. Brunot s'attache à proposer une reformulation des règles orthographiques qui convienne mieux à «la réalité de la langue » et qui rende leur apprentissage plus facile. Prenons l'exemple des règles d'accord du participe passé (envisagées, dans La Pensée et la langue, d'un point de vue quasi exclusivement orthographique). Pour F. Brunot, il ne devrait pas y avoir lieu de distinguer entre les verbes construits avec l'auxiliaire être (= le participe s'accorde avec le sujet) et ceux construits avec l'auxiliaire avoir (= le participe s'accorde avec l'objet direct, s'il en est précédé ; il reste invariable autrement). La règle d'accord du participe passé employé avec avoir, dont C. Marot, F. de Malherbe et C. F. de Vaugelas ont jeté les premières bases mais qui n'a cessé d'être discutée par la suite ${ }^{6}$, a, selon F. Brunot (1922: 325), arrêté «le développement normal de la langue ». En effet, si en ancien français on 
trouve des occurrences du type " (j')ai letres escrites » (ibid. : 324), où escrites - considérée selon F. Brunot comme une épithète ${ }^{7}-\mathrm{s}^{\prime}$ accorde avec letres, à partir du moment où ai écrit a constitué une forme verbale composée, cet accord s'est fait de plus en plus rare, si bien qu'au Xve siècle, on a presque toujours : «j'ai escrit lettres » (ibid.). Ainsi, la règle d'accord du participe passé employé avec avoir va non seulement à l'encontre de l'usage qui avait tendu à s'établir en moyen français, mais est encore contraire à l'« instinct [de] toute personne qui sait la langue » (ibid. : 261) :

"Il importe de marquer combien la règle artificielle qui dure encore violait l'instinct profond qui fait accorder le verbe avec son sujet, et combien, pour cette raison, il a été difficile de la faire accepter. Un homme scrupuleux sur l'orthographe, comme Bossuet, la négligeait constamment, revenant, sans s'en douter, à la vraie règle, savoir de laisser le participe invariable, ou de l'accorder avec le sujet du verbe » (Brunot, $1922: 326$, nous soulignons).

Considérons maintenant la règle d'accord du participe passé des verbes pronominaux, selon laquelle l'accord de ces participes se fait sur le modèle des participes avec avoir. Tout d'abord, comme on l'a vu en 1.1., «les embarras que cause [1']application » de cette règle sont nombreux (Brunot, 1922: 334). Ensuite, cette règle « est contraire à la tradition de la langue » (ibid.), l'usage étant, jusqu'à la fin du xvII siècle, d'accorder le participe des verbes pronominaux avec le sujet du verbe. Cette observation est concordante avec la critique que fait $\mathrm{F}$. Brunot de «la règle artificielle» d'accord du participe passé employé avec avoir. Enfin, cette règle "a le défaut d'être une pure création arbitraire » (ibid.), puisque, comme le suggère F. Brunot, elle ne reposerait sur aucun fondement valable :

«[Les grammairiens de l'Académie] ont considérés [les] verbes [pronominaux] comme s'ils étaient conjugués avec avoir, puisqu'ils ont un objet et que seuls, paraitil, les verbes avec avoir ont le droit d'avoir un objet» (Brunot, 1922: 334, nous soulignons).

La solution proposée par F. Brunot pour reformuler la règle sans changer les formes linguistiques est de renverser la perspective : plutôt que d'étendre la règle d'accord des participes avec avoir aux participes des verbes pronominaux, il est préférable de continuer à considérer, chaque fois que c'est possible, que ces participes suivent la règle d'accord des participes avec être. En effet, en présence de phrases telles que « elles se sont tues, vendues, lavées, taquinées, etc. ", que l'on dise que l'accord se fait avec l'objet direct (le pronom se) ou avec le sujet (le pronom elles), le résultat de l'application de la règle d'accord est identique, le pronom objet étant coréférentiel au sujet de la phrase:

« La règle à enseigner est la suivante : Tout verbe de forme pronominale, que ce verbe soit actif, passif, réfléchi, réciproque, du moment qu'il est construit avec être, accorde, comme les verbes conjugués avec être, son participe avec son sujet :

\begin{tabular}{|l|l|}
\hline Elles se sont tues, repenties, écroulées, & (pronominaux actifs). \\
\hline Elles - vendues, tachées, & (pronominaux passifs). \\
\hline Elles - lavées, habillées, & (réfléchis). \\
\hline Elles - taquinées, averties l'une l'autre & (réciproques)» (Brunot, 1922:335). \\
\hline
\end{tabular}


19 Formulée de la sorte, la règle présente de nombreux avantages. D'un point de vue pédagogique, elle est plus facile à comprendre et à mémoriser, les verbes pronominaux ne constituant plus un cas particulier (ils suivent la règle générale d'accord du participe passé employé avec être). La règle est également plus facile à appliquer, puisqu'elle ne nécessite ni de distinguer entre les verbes suivant qu'ils sont actifs, passifs, réfléchis ou réciproques ou encore "suivant qu'ils sont ou non essentiellement pronominaux » (ibid. : 334), ni d'analyser la fonction du second pronom (se dans les exemples supra). D'autre part, d'un point de vue épistémologique, la règle apparait plus satisfaisante dans la mesure où elle est fidèle à l'histoire de la langue et à l'analyse syntaxique qui prévalait avant l'intervention des grammairiens de l'Académie. Par ailleurs, F. Brunot fait un cas à part des phrases telles «elles se sont adressé des lettres / les lettres qu'elles se sont adressées ", dans lesquelles le participe passé s'accorde avec l'objet direct lorsqu'il en est précédé, mais reste invariable autrement, sur le modèle de la règle d'accord du participe passé employé avec avoir. Pour lui, ce ne sont donc pas l'ensemble (des emplois) des verbes pronominaux qui font exception, mais seulement ceux d'entre eux pour lesquels l'accord du participe passé a changé avec la grammatisation de la langue. En d'autre termes, la solution proposée par F. Brunot est de considérer indépendamment les uns des autres les faits «naturels ", qui suivent des règles partagées par tous les locuteurs et conformes à leur intuition linguistique («l'instinct profond » de la langue), et les faits « artificiels», produits de l'intervention (malencontreuse) des grammairiens sur la langue, qui suivent des règles grammaticales différentes, étrangères aux locuteurs peu instruits ${ }^{8}$ :

«Il n'y a qu'une exception. Si le verbe peut être tourné par le participe avec avoir, et que le pronom se soit, dans l'ancien sens du mot « complément indirect », c.-à.-d. suivant la nouvelle nomenclature, ne soit pas complément d'objet direct, on applique la règle des verbes conjugués avec avoir :

la tâche qu'il s'est donnée ;

les facilités que les deux peuples s'étaient accordées ;

elle s'était croisé les bras ;

ils s'étaient donné mutuellement tous leurs biens.

(1) Les gens du peuple ignorent bien entendu ces règles et accordent avec le sujet; les midinettes disent : le chapeau que je me suis faite. Instinct fort juste. Flaubert, un jour d'oubli, l'a suivi : toutes les injures que l'on s'est dit pour défendre le pur langage » (Brunot, 1922:335, nous soulignons).

D’un point de vue pédagogique, on note la simplicité et la concision de la présentation à laquelle aboutit F. Brunot (par comparaison avec celle d'autres grammaires de l'époque - et même d'aujourd'hui - qui multiplient exceptions et règles particulières). On peut s'étonner que, dans tous les exemples choisis par F. Brunot, le complément d'objet direct soit exprimé sous forme lexicale : ceci s'explique peut-être par la volonté de s'en tenir à des exemples simples, dans lesquels le complément d'objet direct soit facilement repérable. De la même façon, on peut penser que le fait de ne pas mentionner des exemples comme elle s'est arrogé des droits ou elles se sont ri de lui relève d'un souci de simplification de la part de F. Brunot, qui cherche à rendre la règle plus accessible à l'élève ${ }^{9}$. Enfin, en ce qui concerne l'application de la règle, on observe que le premier "test " à opérer, directement inscrit dans la règle même (i.e. vérifier que «le verbe peut être tourné par le participe avec avoir ») ${ }^{10}$, invite à ne travailler que sur des phrases grammaticales (la tâche qu'il s'est donnée / la tache qu'il a donnée) ${ }^{11}$. De même, en l'absence d'indication explicite de F. Brunot, on peut émettre l'hypothèse selon laquelle le second «test» (i.e. vérifier que le pronom se n'est pas complément d'objet direct) n'oblige pas 
davantage l'élève « à choisir entre deux phrases qui n'offrent ni l'une ni l'autre aucun sens » (Brunot, 1909:5;cf. 1.1.) puisque, tout au moins dans les exemples mentionnés par F. Brunot, on voit que le complément d'objet direct se trouve exprimé sous forme lexicale. On peut toutefois regretter que ne soit pas indiqué comment procéder face à des phrases comme nous nous sommes nui (par exemple, en restituant un complément d'objet indirect sous forme lexicale: nous avons nui à cet homme). D'autre part, d'un point de vue épistémologique, un tel partage entre faits "naturels » et "artificiels » permet de relativiser la notion d'exception. Dans une grammaire scolaire comme celle de F.-J.-M. Noël et C.-P. Chapsal ${ }^{12}$, les verbes se douter, s'échapper et se prévaloir sont considérés comme des exceptions car, alors que « le participe des verbes pronominaux formés des verbes neutres est toujours invariable ", pour ces trois verbes, «l'usage veut qu'on fasse toujours accorder le participe avec le second pronom» (1845: 170-171). Dans La Pensée et la langue, en revanche, ces verbes relèvent du modèle général (accord avec le sujet). Toutes les exceptions « véritables » peuvent ainsi être réunies en un seul cas de figure : celui où on peut observer une discordance entre l'intuition des locuteurs et les prescriptions de grammairiens qui seraient intervenus à tort sur l'orthographe de la langue.

\subsection{Règles morphologiques}

21 Dans La Pensée et la langue, le travail de réécriture des règles morphologiques procède d'une volonté de F. Brunot de proposer une description qui soit exacte sur le plan phonétique et qui permette de discerner les "prétendues exceptions » des véritables irrégularités morphologiques. Ses deux exemples favoris sont la règle de formation du féminin des adjectifs qualificatifs, sur laquelle nous ne reviendrons pas, et la règle de formation du futur :

"Donnons un second exemple d'une règle ancienne et fausse: je prendrai celle qu'on enseigne pour la formation du futur. Elle est très simple, sans doute : "A l' infinitif, on ajoute ai, as, a, etc." » (Brunot, 1909 : 106-107).

On constate aisément que cette règle ne convient pas pour les verbes à infinitif en [e] ( -er) comme aimer, jeter, semer, etc. En effet, si, d'un point de vue strictement graphique, aimer et -ai mis bout à bout donnent bien la forme attendue (aimerai), d'un point de vue

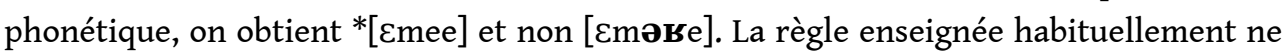
vaut ainsi que pour l'écriture, elle ignore le « changement réel, sensible à l'oreille, que l'élève peut avoir remarqué » (Brunot, 1909 : 105). De plus, cette règle ne permet pas de rendre compte des verbes pour lesquels les différences phonétiques entre la forme infinitive et le radical du futur s'accompagnent d'une modification orthographique de ce dernier : jeter + ai donne jeterai et non jetterai, semer + ai donne semerai et non sèmerai. Pour palier ces deux difficultés, F. Brunot propose la règle suivante (qui est celle généralement enseignée aujourd'hui) :

«La vérité est que, depuis longtemps, le futur des verbes en $e$ (infinitif er), c'est-àdire de l'immense majorité des verbes, se forme de la lère personne du singulier $d u$ présent de l'indicatif, suivie de l'r de l'infinitif, et des terminaisons ai, as, a, etc.» (Brunot, 1909: 107).

Cette règle présente l'avantage d'être exacte à la fois d'un point de vue orthographique et d'un point de vue phonétique ${ }^{13}$, mais aussi de ramener au statut de formes régulières les formes jetterai, sèmerai, etc., jusque-là considérées comme des exceptions. Plus 
généralement, c'est la présentation usuelle de l'ensemble des formes de futur qu'il convient de revoir :

«Au lieu de me contenter d'une fausse règle toute apparente, et de présenter le reste comme « exceptions » il me semble qu'il y aurait quelque chose de gagné pour la grammaire et pour l'éducation générale, si l'on disait: $\mathrm{Il}$ y a trois espèces de futurs dans notre langue: $1^{\circ}$ de vieilles formes contractes qui ne s'expliquent que par le latin : mourrai, verrai ; $2^{\circ}$ des futurs formés de l'infinitif : finirai, rendrai $; 3^{\circ}$ des futurs modernes formés du présent de l'indicatif auquel on ajoute $r$ de l'infinitif, puis la terminaison » (Brunot, 1913a : 135, nous soulignons).

Si F. Brunot cherche bien à donner une description en synchronie du français moderne, il ne s'interdit pas pour autant de recourir à des éléments d'histoire de la langue lorsque cela s'avère pertinent, c'est-à-dire lorsque ces éléments peuvent être utilisés à des fins explicatives et/ou de classification. Il apparait ainsi qu'on peut séparer les formes $d u$ futur du français moderne en trois groupes distincts, qui correspondent chacun à trois moments de formation différents dans l'histoire de la langue, que l'on peut présenter schématiquement comme suit :

- en latin vulgaire, le futur est exprimé à l'aide d'une périphrase composée de l'infinitif du verbe suivi de habere conjugué au présent de l'indicatif; cette périphrase a ensuite évolué phonétiquement jusqu'à aboutir aux formes synthétiques que l'on rencontre dans les premiers textes français : salvare habeo > * salvaraio > salverai « je sauverai »; ${ }^{\text {prendre habeo > }}$ *prendraio > prendrai « je prendrai »;

- en ancien français, le futur des nouveaux verbes est formé de l'infinitif auquel on ajoute les désinences du verbe avoir, "devenues de véritables flexions temporelles et personnelles "

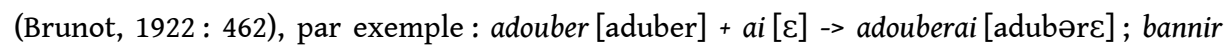

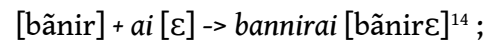

- en français classique et moderne, le futur des nouveaux verbes est formé du radical du présent de l'indicatif auquel on ajoute $r+a i$, as, a, etc., par exemple : (je) sombre [s刀̃bьə] $+r$

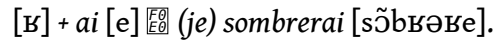

De plus, on observe que, pour les verbes déjà existants, divers phénomènes de réfection se produisent :

- pendant toute la période de l'ancien et du moyen français jusqu'au milieu du XVII siècle, « un certain nombre de verbes refirent leur futur sur le type contracté, soit que l'e muet eût peine à se maintenir, soit que l'existence des futurs contractés exerçât une action analogique » (Brunot, 1922 : 462) : à côté de donerai, on rencontre, les formes donrai et dorrai, qualifiées de «monstres » par C. F. de Vaugelas; inversement, les futurs de type 2 (formés sur l'infinitif) exercent leur action sur les futurs de type contracté: assaudray est progressivement remplacé par assailliray ; en revanche verrai, un temps en concurrence avec voirai, se maintient;

- à partir du moment où «on eut le sentiment que le futur était apparenté à l'indicatif présent » (Brunot, 1913: 709), on observe une influence des formes de présent sur les futurs de type 1: concurrence entre orray (type 1), ouiray (type 2) et oiray (forme refaite sur le présent); de type 2 : concurrence entre tressaillirai et tressaillerai (le type 2 se maintient), entre cueillirai et cueillerai (la forme refaite sur le présent s'impose); et même de type 3 : alignement phonétique des futurs de jeter et semer sur les formes du présent jette, sème (base tonique en $[\varepsilon])$.

L'objectif de F. Brunot n'est pas ici de plaider en faveur d'un enseignement de la grammaire historique à l'école et au collège ${ }^{15}$, mais de montrer que la leçon de grammaire gagne à être fondée sur deux principes fondamentaux. Le premier est qu'il 
faut "écarte[r] l'apparence orthographique pour chercher la réalité linguistique " (Brunot, 1913a : 134). Comme on sait, au cours de la période du moyen français, le $r$ de l'infinitif des verbes de la première conjugaison a cessé de se prononcer; toutefois, l'orthographe étant restée identique (ou quasi identique), on a pu continuer à dire que le futur des verbes de la première conjugaison était issu de l'infinitif. Seule l'analyse de la prononciation permet de faire apparaitre que « la règle moderne pour la formation du futur [...] est double " (Brunot, 1922: 464) : pour les verbes en [в], formation sur l'infinitif ; pour les verbes en [e], formation sur le présent. Le second principe est qu'il faut « regarder la langue comme elle est, comme les recherches linguistiques ont montré qu'elle est, c'est-à-dire comme une chose en évolution» (Brunot, 1913a: 133). On peut ainsi faire apparaitre que trois types d'infinitifs coexistent dans la langue moderne : des futurs réguliers d'une part, formés sur l'infinitif ou le présent ; des futurs irréguliers d'autre part, qui sont « des faits anciens qui n'ont pas évolué » (ibid.), issus de formes régulières du latin populaire. En relativisant de la sorte le statut des formes considérées jusque-là comme des exceptions, on aboutit à une description plus satisfaisante ; en effet :

"Chose précieuse entre toutes dans une éducation, les faits, au lieu de se heurter dans une mêlée chaotique et contradictoire apparaissent dans un ordre qui est en même temps historique et pédagogique " (Brunot, 1913a : 135, nous soulignons).

\subsection{Règles syntaxiques}

F. Brunot (1909 : 108) note que, «en général, les règles de syntaxe renferment moins d'erreurs positives et matérielles » que les règles de morphologie. Selon lui, le principal défaut des règles syntaxiques établies par ses prédécesseurs tient - on l'a souligné précédemment - à leur étroitesse, leur rigidité : la plupart du temps, ces règles ne permettent pas de rendre compte de l'usage, de la multiplicité et de la complexité des réalisations en discours. Ici, l'objectif, ou pour mieux dire l'objet, de F. Brunot n'est pas de donner de nouvelles règles : l'entreprise en soi est vaine, la plus grande partie des emplois échappant à toute tentative de formalisation. Tout au plus peut-on établir quelques "principes généraux ». À travers les critiques de F. Brunot apparait ainsi en filigrane un plaidoyer en faveur d'une syntaxe qui accorde une place première au sens, une syntaxe "souple", au service de l'expression et de l'expressivité. Considérons l'exemple de quelques règles d'emploi du subjonctif: "La règle générale des langues romanes, dit F. Brunot, est qu'on met le subjonctif après proposition négative " (Brunot, 1922 : 522). Or l'application «brutale » d'une telle règle, c'est-à-dire sans tenir " aucun compte du sens" syntaxique (ibid.: 523), fait courir le risque de priver le locuteur de la possibilité d'exprimer une nuance de sens. Si l'on dit «il ne peut admettre que sa femme est morte ", la mort de la femme est avérée ; si l'on emploie au contraire le subjonctif, sa mort n'est pas certaine. De la même manière, il n'est pas équivalent d'employer l'indicatif ou le subjonctif après une proposition interrogative "pensez-vous que cela peut/puisse convenir? ». Plus grave, un emploi «mécanique » du subjonctif peut conduire à des "illogismes » (ibid. : 522). Ainsi, après ne pas douter, la subordonnée est au subjonctif alors même qu'une certitude (ou au moins l'absence de doute) est exprimée : «je ne doute pas que cela (ne) soit vrai », «je ne doute pas qu'il vienne", etc. Selon F. Brunot, le problème est qu'ici le mode subjonctif s'introduit "dans des phrases où la pensée ne le justifie pas et où seule l'analogie des formes l'amène ", de sorte qu'il n'exprime plus une modalité, "mais n'est qu'une forme de 
subordination" (ibid. : 520-521). F. Brunot défend ainsi l'idée que l'imposition stricte de l'emploi du subjonctif dans certaines constructions syntaxiques aurait provoqué un mouvement d'analogie faisant naitre des "discordances entre le langage et la pensée ", c'est-à-dire entre les formes et leur "valeur réelle " (ibid. : 523-524) - ce qui aurait par là même conduit à abandonner petit à petit l'emploi de ce mode ${ }^{16}$. Il faut donc distinguer entre deux types de mouvements d'évolution de la langue : ceux qui auraient une cause "artificielle ", " extérieure à la pensée " (l'imposition de règles "rigides " par les grammairiens) et ceux qui seraient proprement "naturels». Alors que la généralisation de l'emploi $d u$ subjonctif après les propositions négatives et interrogatives relèveraient du premier type, la généralisation de ce mode après les verbes de sentiment (j'enrage qu'il ait signé sans me consulter) relèveraient du second type. C'est en tout cas ainsi que nous interprétons ce passage :

«En somme, comme partout, le subjonctif s'est imposé au verbe qui exprime l'objet-action après les verbes de sentiment. Et $\underline{i l \text { ne faut pas voir là un fait de }}$ l'autorité ou une extension mécanique. Il semble que de plus en plus, par une sorte d'unification, quand la phrase se construit en subordination, l'objet qui exprime en fait la cause et l'origine du sentiment s'incorpore de façon plus intime à l'ensemble. La construction devient de plus en plus synthétique, pendant que, grâce aux phrases décomposées, on peut isoler d'une part le fait, de l'autre le sentiment qu'il produit et qu'on éprouve » (Brunot, 1922 : 556, nous soulignons).

Qu'en est-il maintenant des exemples qui dérogent aux règles syntaxiques que l'on vient de mentionner? Pour F. Brunot, il ne s'agit ni d'« exceptions », ni de «fautes". Toutes les formes linguistiques, si tant est qu'elles soient motivées par les besoins de la "pensée ", de l'expression, ont (potentiellement) droit de cité. Il existe un emploi légitime du subjonctif après proposition négative :

«En langue moderne, la pensée tend à se dégager des contraintes, ce qui est une façon de suivre la vraie et bonne tradition. A vrai dire, on rend ainsi au subjonctif sa valeur: Malheur à l'homme qui, dans les premiers moments d'une liaison d'amour, ne croit pas que cette liaison doit être éternelle (B. CONST., Ad., 29). Allusion est faite à une croyance ferme » (Brunot, 1992 : 523, nous soulignons).

Et même après un verbe de sentiment: "je me réjouis de ce que ce bon petit va mieux ", «il se plaint de ce qu'elle l'a trompé ", etc. ${ }^{17}$ L'exemple de B. Constant est mobilisé dans l'argumentation de F. Brunot pour montrer que la langue tend à s'affranchir des contraintes qui lui sont imposées de l'extérieur ; les exemples cités à l'instant témoignent quant à eux de l'existence d'un conflit dans l'évolution interne de la langue (tendance à la synthèse versus expression de la modalité). Dans les deux cas, ces exemples - et encore de nombreux autres, concernant notamment l'emploi des temps du subjonctif - permettent à $\mathrm{F}$. Brunot de mettre en lumière ce qu'il appelle une « loi de langage»:

«La loi générale est que la modalité l'emporte sur la relation » (Brunot, 1922 : 710). «C'est toujours la même loi qui agit sur le langage. Les contraintes imposées pour marquer les relations logiques cèdent à un instinct supérieur, qui pousse à exprimer la modalité sous laquelle le fait apparait à l'esprit. C'est là l'essentiel pour la pensée. Le sens domine les mécanismes syntaxiques" (ibid.: 898, nous soulignons).

L'objectif de F. Brunot est ainsi de discuter l'adéquation des règles syntaxiques avec les lois (qui dirigent la marche) du langage et de montrer que la syntaxe ne se réduit pas à de simples mécanismes grammaticaux. 


\section{Qu'est-ce qu'une « vraie règle » de grammaire selon F. Brunot ? Qu'est-ce que la « grammaire vraie » ?}

31 L'entreprise de critique et de réécriture des règles de la "grammaire dogmatique " menée par F. Brunot dans La Pensée et la langue doit être replacée dans le contexte social et idéologique de la première moitié $\mathrm{du} \mathrm{xx}^{\mathrm{e}}$ siècle. $\mathrm{F}$. Brunot $\mathrm{y}$ fait figure de linguiste militant : il s'oppose au discours, alors largement en circulation, selon lequel il y aurait une «crise du français» et maintient qu'il y aurait seulement une «crise» de l'enseignement de la langue. F. Brunot plaide pour une rénovation de la pédagogie traditionnelle. Il remet en cause un enseignement qu'il estime fondé sur « l'idée que les langues s'enseign[ent] théoriquement par règles et par chapitres, en récitant des formules et des exemples » (Brunot, $1909: 38)$ et propose au contraire d'avoir recours à une "méthode inductive» (ibid.: 71), qui part de l'observation des productions particulières des élèves pour les amener à dégager progressivement ce qu'il y a de commun entre elles, et à formuler d'eux-mêmes la règle :

«Se produisant à ce moment, quand elle [la règle] aura été découverte par un effort personnel, ne voit-on pas qu'elle aura une autre réalité, une autre autorité pour l'enfant que si on a commencé par elle, que si on l'a énoncée toute faite » (Brunot, 1909 : 135)?

La "méthode inductive " permet ainsi de renouer avec la pratique quotidienne des élèves. Conduite de cette manière, la leçon de grammaire est au service de l'enseignement de la langue, elle remplit de nouveaux objectifs pédagogiques :

«La langue vit en lui [l'enfant], il la parle, il la sait, au moins par à peu près. Mon travail consiste à corriger son usage là où il est défectueux, sans doute, mais surtout à amener l'enfant par un travail infiniment fécond, non seulement au point de vue grammatical, mais d'une façon générale, pour son éducation psychologique, à prendre conscience de son langage » (Brunot, 1913a : 137, nous soulignons).

Pour F. Brunot (1922 : XXIII), il ne s'agit donc pas simplement d'apprendre à l'élève à écrire correctement mais de le conduire "à voir clair dans sa pensée, à analyser ce qu'il veut dire et écrire", afin qu'il soit capable d'exprimer ses idées le plus judicieusement possible.

Le deuxième aspect remarquable de la réflexion de F. Brunot, à côté de la place à donner à la grammaire dans l'enseignement de la langue, est le rôle qu'il faut accorder à l'histoire de la langue dans la description grammaticale. En effet, la grammaire historique

«a [...] commencé à faire pénétrer dans quelques cerveaux une conception nouvelle de la règle grammaticale; elle $\mathrm{y}$ a introduit l'idée du mouvement, elle en a ainsi ruiné l'absolutisme, car, en faisant connaître l'âge et les origines des dogmes, elle a permis d'en mesurer la valeur véritable » (Brunot, 1922 : XII, nous soulignons).

Pour F. Brunot (1913a : 133), faire de la grammaire sans prendre en considération le fait que la langue est « une chose en évolution », c'est se leurrer sur la nature des règles de la langue :

«Le langage est muable. Il n'y a personne, aucune autorité, ni l'Académie, ni l'Université, qui à un moment donné ait reçu du ciel les règles de la langue, règles éternelles et absolues; c'est là de la fantaisie, non de la réalité. La règle n'est pas une vérité révélée » (Brunot, 1913a: 132, nous soulignons).

L'entreprise de critique des règles menée par $\mathrm{F}$. Brunot vise au contraire à montrer que celles-ci sont le produit de l'histoire de la langue, en même temps que de l'histoire des 
descriptions auxquelles elle a donné lieu. Cette observation explique sans doute certaines ambigüités du métadiscours de $\mathrm{F}$. Brunot, qui désigne par le même terme de règle des objets aux statuts épistémologiques différents: les "règles artificielles » imposées par certains grammairiens; les "principes généraux » sur lesquels reposent la (morpho)syntaxe de la langue (accord de l'adjectif épithète, place par rapport au nom) ; les « lois du langage " qui, sur le modèle des lois phonétiques de la linguistique historique et comparée, rendent compte des évolutions morphologiques (formation du futur) et de la manière dont se règlent les conflits entre syntaxe et sémantique (emploi du subjonctif). Le recours à l'histoire de la langue permet ainsi de satisfaire l'ambition de généralité de F. Brunot, qui cherche à montrer qu'il n'y a pas d'« exception » dans la langue, qu'il n'y a pas de fait qu'on ne puisse expliquer : les exceptions orthographiques ne sont pour lui que des "anomalies", des "faits anormaux" dont on devrait se débarrasser; les exceptions morphologiques sont soit des formes régulières mal décrites, soit des formes irrégulières issues d'anciennes formes régulières; les exceptions aux règles de syntaxe (de même que certaines « fautes » condamnées par les puristes) sont motivées par des nécessités d'expression et ne sont de ce fait pas moins légitimes que les autres réalisations syntaxiques.

À partir de cette étude sur la règle et l'exception chez F. Brunot, nous pouvons voir émerger certains aspects saillants de l'épistémologie de l'auteur, qui constituent pour nous autant de pistes de travail à explorer. On peut évoquer en premier lieu la position (apparemment) paradoxale dans laquelle se place F. Brunot (1913a : 133), qui conçoit la grammaire comme une science de la nature dont on peut dégager les lois, en même temps qu'il soutient que les langues sont "des faits sociaux" - position qu'il faut replacer dans la réflexion épistémologique au tournant du $\mathrm{XIX}^{\mathrm{e}}$ et $\mathrm{du} \mathrm{Xx}^{\mathrm{e}}$ siècle dont l'enjeu, pour les sociologues (É. Durkeim, L. Lévy-Bruhl) et linguistes formés au positivisme, est de défendre la possibilité de faire « des sciences de l'esprit qui ne soient pas normatives» (Auroux, 1994: 296). Chez F. Brunot, cette idée que l'on peut " décri[re] des irrégularités dans le réel, même dans le domaine de l'histoire » (ibid.) est justement rendue possible par la prise en compte de l'histoire dans la description: l'histoire de la langue d'une part ${ }^{18}$, mais aussi l'histoire de la société, dont on peut apprécier l'impact sur la langue à travers l'analyse de l'évolution des usages. On retrouve chez $\mathrm{F}$. Brunot une conception de la langue comparable à celle de A. Darmesteter (après A. Schleicher), celle d'un organisme vivant mu par des forces internes ${ }^{19}$. À cela s'ajoute l'idée qu'il y a une vérité de la langue à chercher dans le langage du peuple (« l'instinct populaire »), topos de l'époque dont l'évocation répond, d'un auteur à l'autre, à des enjeux différents, et qui est à replacer plus largement dans le cadre de la réflexion sur (ce qu'on peut appeler) les anomalies linguistiques : moteur de l'évolution de la langue pour $\mathrm{H}$. Frei; reflet du fonctionnement du système linguistique pour $\mathrm{C}$. Bally ; mode d'accès privilégié à l'inconscient pour J. Damourette et É. Pichon. On peut évoquer en second lieu la critique implicite de l'opposition langue/ parole par F. Brunot (qui ne cite jamais F. de Saussure): de ce point de vue, ses observations sur la rigidité des règles sont concordantes avec son travail de remise en cause des catégories grammaticales car, pour lui, « les éléments linguistiques n'ont pas une valeur constante" (1922: XII). F. Brunot refuse aussi bien le caractère artefactuel des règles des grammairiens que de la "langue idéale " qu'ils décrivent. Tout son métadiscours est émaillé de termes qui dessinent un imaginaire du "naturel», du « réel » de la langue. L'originalité de son travail est d'avoir montré qu'il n'y a pas de solution de continuité entre l'histoire des idées linguistiques, l'histoire des outils 
linguistiques (grammaires, dictionnaires) et l'histoire de la langue. Pour lui, faire de la grammaire en linguiste, c'est réfléchir sur le corpus des règles fournies par la tradition et démêler ce qui est conforme aux lois du langage de ce qui résulte de l'action des grammairiens sur la langue. Cela ne signifie pas que F. Brunot s'oppose au fait de normer la langue : selon lui, le rôle du (bon) grammairien est de venir entériner l'usage général, d'accueillir les « créations justifiées et [les] changements nécessaires » (1922: XIII) et de guider ainsi, avec discernement, la marche du langage.

\section{Références primaires}

ARNAULD, A. \& LANCELOT, C. (1676) [1660]. Grammaire générale et raisonnée. Paris : Pierre Le Petit.

BRUNOT, F. (1905). La Réforme de l'orthographe : lettre ouverte à M. le ministre de l'Instruction publique.

Paris : Armand Colin.

- (1906). « La simplification de l'orthographe ». La Revue de Paris 6, p. 1-39 et p. 383-416.

- (1909). L'Enseignement de la langue française : ce qu'il est, ce qu'il devrait être dans l'enseignement primaire. Paris : Armand Colin.

- (1913a). « La méthode positive en grammaire ». In Croiset, A. et al., La Méthode positive dans l'enseignement primaire et secondaire. Paris : Félix Alcan, p. 125-150.

- (1913b). Histoire de la langue française des origines à 1900, t. IV, La langue classique (1660-1715). Paris : Armand Colin.

- (1920). « Le renouvellement nécessaire des méthodes grammaticales ». Revue universitaire 8-2, p. $161-178$.

- (1921). « Le renouvellement nécessaire des méthodes grammaticales : deuxième article ». Revue universitaire 9-1, p. 21-39.

- (1965) [1922]. La Pensée et la langue : méthode, principes et plan d'une théorie nouvelle du langage appliquée au français, $3^{\mathrm{e}}$ éd. revue ( $3^{\mathrm{e}}$ tirage). Paris : Masson.

NOËL, F.-J.-M. \& CHAPSAL, C.-P. (1845) [1823]. Nouvelle Grammaire française, $38^{\mathrm{e}}$ éd. revue avec soin et augmentée, Paris : Maire-Nyon/Roret/Hachette/Delalain.

\section{Références secondaires}

AUROUX, S. (1994). «Remarques sur l'histoire philosophique du concept de "norme" et sur l'histoire des sciences du langage ». In Kasbarian, J.-M. (éd.), Genèse de la (des) norme(s) linguistique(s). Aix-en-Provence : Publications de l'université de Provence.

BESSE, H. (1995). « Ferdinand Brunot, méthodologue de l'enseignement de la langue française ». Histoire, Épistémologie, Langage 17-1, p. 41-74.

BLANCHE-BENVENISTE, C. (1990). « Grammaire première et grammaire seconde : l'exemple de en ». Recherches sur le français parlé 14, p. 51-73. 
CHERVEL, A. (1977). Et il fallut apprendre à écrire à tous les petits français : histoire de la grammaire scolaire. Paris : Payot.

CHEVALIER, J.-C. (1991). « Ferdinand Brunot (1860-1937) La Pensée et la Langue ». In Huot, H. (dir.), La Grammaire française en comparatisme et structuralisme, 1870-1960, Paris : Armand Colin, p. 73-114.

ELALOUF, A. (2012). « La notion de "grammaire seconde" : tentative de reconstruction épistémologique ". In actes du CMLF 2012 - III ${ }^{e}$ Congrès mondial de linguistique française. EDP sciences. En ligne : http://dx.doi.org/10.1051/shsconf/20120100328.

\section{NOTES}

1. Notre objet ici n'est pas de traiter du rapport de F. Brunot à la norme linguistique et de ce qu'il considère comme étant des formes «incorrectes" ou "ano(r)males» (i.e. des formes qui dérogent à la règle et qui ne sont pas jugées valides par le grammairien). Ce sujet mériterait une étude en soi, dont nous évoquerons quelques aspects en conclusion.

2. Le corpus est constitué non seulement de l'intégralité de La Pensée et la langue, mais encore d'un ensemble de textes plus anciens (Brunot, 1905, 1906, 1909, 1913a, 1920, 1921) dans lesquels on peut trouver des développements sur la notion de « règle grammaticale » et/ou des observations éparses sur telle ou telle règle particulière (voir les références primaires en bibliographie).

3. Pour A. Chervel (1977 : 264), F. Brunot « est le premier à avoir aussi clairement discerné la relation intrinsèque qui unit l'orthographe et la grammaire scolaire» .

4. A. Arnauld et C. Lancelot (1676:38-39) remarquent par exemple : « On dit bien fers au pluriel ; mais c'est pour signifier des chaisnes, \& non seulement une partie du metail appelé fer. »

5. Il faut ajouter le rôle joué par la disparition des formes toniques de pronoms sujets dans ce processus.

6. F. Brunot (1913b : 925-938) retrace le détail de cette histoire dans l'Histoire de la langue, des origines à 1900. Il mentionne notamment les objections de F.-S. Régnier-Desmarais et de C. Buffier, qui plaident en faveur d'une extension de l'invariabilité du participe.

7. Nous dirions plutôt qu'il s'agit d'un attribut de l'objet direct.

8. Cette distinction est théorisée par C. Blanche-Benveniste (1990) à travers l'opposition entre faits de " grammaire première " (usages connus de tous, dès avant l'âge de l'école primaire) et faits de "grammaire seconde » (usages qui ne sont maitrisés que par les locuteurs ayant atteint un certain degré de scolarisation). Ses exemples sont toutefois plutôt empruntés à la morphosyntaxe qu'à l'orthographe (pour cause, avant l'école primaire, on ne sait pas encore écrire). Pour une analyse, voir A. Elalouf (2012).

9. La phrase elle s'est arrogé des droits est évoquée dans l'argumentation mais n'est pas reprise comme exemple illustrant la règle, sans doute parce que la substitution de l'auxiliaire être par l'auxiliaire avoir n'y est plus possible (jusqu'au Xvi , on peut trouver des occurrences du type : «Il [le Roi] ne peult arroger tant d'honneur a beaucoup pres aux dessusdictz ses deleguez, comme il en derogue a sa majesté »; source : TLFi). La phrase elles se sont ri de lui est seulement évoquée en note de bas de page. Le pronom se n'y étant pas analysable, ce cas est laissé de côté.

10. F. Brunot note qu'on trouve déjà dans la grammaire de N. Landais (1835) l'observation suivante: «Cette règle (celle des participes construits avec être)... est applicable aux participes des verbes réfléchis et réciproques... quand l'analyse ne permet pas de remplacer l'auxiliaire être par avoir. " (cité par Brunot, 1922 : 335).

11. Dans le cas où la phrase obtenue est agrammaticale (nous nous sommes attaqués à plus fort que nous / "nous avons attaqués à plus fort que nous), on sait que l'exemple relève de la règle d'accord des participes employés avec être, sans qu'il soit nécessaire d'analyser la fonction du pronom nous. 
12. La « [grammaire] a peu près adoptée universellement pendant un demi-siècle, et qui a été, en fait de langue française, la Bible de plusieurs générations d'écoliers " (Brunot : 1909 : 43).

13. Sauf dans le cas d'un verbe comme compléter, ou l'on écrit je complète versus je compléterai, mais où l'on prononce bien dans les deux cas un $[\varepsilon]$. Il s'agit là selon F. Brunot (1909: 30-31) d'une inconséquence orthographique dont il faudrait se débarrasser.

14. Pour les verbes de la première conjugaison, il faut noter que «par apophonie, la voyelle de l'infinitif [est] modifiée, en devenant atone» (Brunot, 1922: 462). F. Brunot (1913b: 709) remarque que « c'était là une apophonie commune dans la langue, tout à fait semblable à celle de berger, bergerie ».

15. Un tel enseignement, remarque F. Brunot (1913a: 134), serait incompréhensible pour des élèves qui ne connaissent pas, ou à peine, le latin : «Les engager dans ce dédale, c'est risquer de les perdre, et je suis bien revenu, je le confesse, des idées ambitieuses que j'ai eues autrefois sur ce point ».

16. F. Brunot n'évoque pas les confusions possibles entre les formes d'indicatif et de subjonctif qui sont homophones voire homonymes. Peut-être ces confusions ont-elles joué un rôle dans le processus qu'il décrit.

17. F. Brunot fait ici de l'utilisation de de ce que une caractéristique de la «langue populaire moderne »; il suggère néanmoins que l'on pourrait profiter "de cette ressource précieuse » (Brunot, 1922 : 548). Cet emploi existait d'ailleurs à l'époque classique : «Je ne m'estonne pas de ce que vos grands Ouvriers font tomber des Perles liquides de ses doigts (РЕTIT, Dial. Sat., 56)» (ibid.).

18. J.-C. Chevalier (1995 : 97) fait remarquer que, chez F. Brunot, «par un bizarre retournement du couple saussurien, la synchronie est le lieu de tous les débordements et la diachronie le fil de toutes les coutures nécessaires ».

19. L'objet de la grammaire, dit F. Brunot (1922: XII), « est de donner une idée de ce qu'est le langage, avec ses nuances, ses inconséquences, mêlée perpétuelle d'éléments que des forces naturelles poussent vers la confusion, pendant que d'autres organisent et distinguent, enchevêtré, indécis, complexe comme la nature, et non réduit, simplifié, ordonné, aligné comme la fausse science ».

\section{RÉSUMÉS}

Cette étude est une contribution à l'analyse historique et épistémologique des travaux de F. Brunot. Nous y examinons les différents aspects de sa réflexion sur les notions de "règle grammaticale » et d'« exception » ainsi que les incidences de cette réflexion sur sa pratique de grammairien dans La Pensée et la langue (1922). Selon F. Brunot, la plupart des règles de grammaire établies par ses prédécesseurs souffrent de nombreux défauts : d'un point de vue pédagogique, elles donnent lieu à un enseignement qui n'aurait que peu d'incidence sur les productions des élèves et qui tendrait à fausser leur rapport à la langue; d'un point de vue épistémologique, elles décriraient de façon inadéquate les faits linguistiques et iraient souvent à l'encontre de l'évolution «naturelle " de la langue. L'analyse de la manière dont ces règles sont reformulées dans La Pensée et la langue met en évidence la nécessité qu'il y a pour F. Brunot de regarder la langue comme "une chose en évolution» et d'établir un partage entre les faits «naturels» et "artificiels», qui résulteraient de l'intervention malencontreuse des grammairiens sur la langue. Par là même, il montre que les règles sont le produit de l'histoire de 
la langue en même temps que de l'histoire des descriptions auxquelles elle a donné lieu, et que les exceptions n'existent pas en ce sens qu'il n'y a pas de fait linguistique qu'on ne puisse expliquer.

This paper is a contribution to the historical and epistemological analysis of Ferdinand Brunot's work. We discuss various aspects of his reflection on the notions of "grammatical rule" and "exception" as well as the impact of this reflection on the grammatical description in La Pensée et la langue (1922). According to Brunot, there are many faults in most of the grammatical rules established by his predecessors: from a pedagogical point of view, they give rise to teaching that only has a limited impact on what pupils produce and tends to distort the relation to language; from an epistemological point of view, they describe linguistic facts inadequately and often run counter to the "natural" evolution of language. The analysis of the way these rules are reformulated in La Pensée et la langue highlights Brunot's thesis considering the language as "an evolving thing" and establishing a division between "natural" facts and "artificial" facts, which is the result of grammarians' unfortunate intervention in language. Thereby, he shows that rules are the product of the history of language as well as the history of grammatical descriptions, and that exceptions do not exist in the sense that there is no linguistic fact that cannot be explained.

\section{INDEX}

Keywords : Ferdinand Brunot, French grammar, rule, exception, history of language

Mots-clés : Ferdinand Brunot, grammaire française, règle, exception, histoire de la langue

\section{AUTEUR}

AURÉLIA ELALOUF

HTL (UMR 7597), Université Sorbonne Nouvelle - Paris 3 